\title{
Género y trabajo: experiencia de las prestadoras de las casas de salud de mujeres indígenas de Guerrero y Oaxaca
}

\author{
Gender and work: the experience of providers of health services \\ to indigenous women from Guerrero and Oaxaca
}

Claudia Isabel Amaya-Castellanos ${ }^{1}$

Blanca Pelcastre-Villafuerte ${ }^{1}$

Margarita Márquez-Serrano ${ }^{1}$

Clara Juárez-Ramírez ${ }^{1}$

${ }^{1}$ Instituto Nacional de Salud Pública. Avenida Universidad 655, Colonia Santa María Ahuacatitlán. 62100 Cuernavaca Morelos México

caguiamaya@hotmail.com
Abstract The scope of this paper was to identify the role of gender for women as workers, through the perception and discourse of health service providers at the 'Health Centers for Indigenous Women' ("Casas de Salud de las Mujeres Indigenas") in Ometepec, Guerrero and Matías Romero, Oaxaca, Mexico. It is a qualitative study, based on a secondary analysis of the interviews conducted as part of the "Rescatando la experiencia de la Casa de la Mujer Indígena: sistematización y evaluación del proceso" project. A strong sense of the value of work and a strong commitment towards the community were identified. Guilt appears as the result of tension between work outside the home and the responsibilities as mothers and wives. The possibility of helping other women is a source of gratification that dissipates these conflicts; prestige and recognition are added to the benefits of their role. The labor experience of female workers in the "Casas de Salud de las Mujeres Indígenas" of Matías Romero, Oaxaca and Ometepec, Guerrero, described in this paper, reveals that it has very specific characteristics, which are difficult to replicate in other regions of the country. In future studies it is necessary to consider the different contexts of vulnerability.

Key words Indigenous women, Gender, Labor process
Resumen Objetivo. Identificar, a través del discurso de prestadoras de servicio de las Casas de Salud de las Mujeres Indígenas de México en Ometepec, Guerrero y Matías Romero, Oaxaca, el papel de género en el desempeño de su rol como trabajadoras. Metodología. Estudio cualitativo, basado en un análisis secundario de las entrevistas realizadas como parte del proyecto "Rescatando la experiencia de la Casa de la Mujer Indígena: sistematización y evaluación del proceso". Resultados. Se identificó un importante valor al trabajo y compromiso hacia la comunidad. La culpa aparece como producto de la tensión entre el trabajo fuera del hogar y las responsabilidades como madres y esposas. La posibilidad de ayudar a otras mujeres es fuente de gratificación que disipa estos conflictos; el prestigio y el reconocimiento se suman a las ganancias de su rol. Conclusiones. La experiencia laboral de las mujeres trabajadoras en la "casa de la mujer indígena" de Matías Romero, Oaxaca, y Ometepec, Guerrero, descrita en este trabajo muestra que tiene características muy específicas de difícil replicación en otras regiones del país. En estudios futuros es necesario considerar los diferentes contextos de vulnerabilidad.

Palabras clave Mujeres indígenas, Género, Proceso de trabajo 


\section{Introducción}

La incursión de las mujeres en el ámbito laboral a nivel mundial se ha venido incrementando; entre 1930 y 1970 la población femenina económicamente activa creció a un ritmo anual de 5.96\%, comparado con el $2.29 \%$ de los hombres. En América Latina el incremento ha sido muy importante; en México, se habla de un incremento gradual de $21 \%$ en 1979 a $34 \%$ en $1995^{1}$. Las mujeres se han incorporado a la actividad económica preferentemente en el sector terciario, en ocupaciones altamente feminizadas, conformando espacios sexualmente segregados como: secretarias, maestras, meseras, enfermeras y trabajadoras no calificadas en los servicios personales.

En el caso de las mujeres indígenas por las situaciones precarias en las que viven, también han tenido que incorporarse a la actividad económica fuera del contexto indígena, ya que al interior de su estructura social, comúnmente su mano de obra es utilizada en la elaboración de artesanías o en el cuidado y recolección de cultivos. Según el censo para población indígena realizado en 2000, se reporta una participación de las mujeres indígenas de $25.6 \%$ frente a un $70.8 \%$ de los hombres. Su participación se ha centrado mayoritariamente en actividades agropecuarias (47\%) y algunas incursiones en el comercio $(12 \%)$; otras se dedican a la elaboración de prendas de vestir $(8 \%)$ y un $8 \%$ más trabaja en el servicio doméstico. Muchas de ellas también se han incorporado a labores de tipo comunitario, gestionando servicios básicos en educación, salud, infraestructura de vías y ambiente, favoreciendo el bienestar social de sus pueblos ${ }^{2}$.

Sin embargo, se hace difícil identificar claramente la participación económica de éstas, ya que para ellas existe la dificultad en delimitar "el trabajo", "la ayuda" y el "trabajo no remunerado", aun cuando estas actividades sean diferentes a las domésticas ${ }^{3}$. En este punto es importante resaltar que el incremento de la fuerza laboral femenina, tanto en comunidades indígenas como no indígenas, se ha hecho evidente a partir del trabajo extra-doméstico en el que las mujeres han ido incursionando gradualmente; sin embargo, la existencia de un trabajo doméstico ha sido permanente y casi inherente a la condición de ser mujer.

En México, de acuerdo a la Encuesta Nacional de Empleo (ENE) de 1993, más de 80\% de las mujeres mayores de 12 años de edad realiza algún tipo de labor doméstica, comparado con $25 \%$ de los hombres ${ }^{1}$. Sin embargo, este rol de la mujer no ha sido reconocido como forma de contri- bución al sistema económico de las familias, quizá porque históricamente han prevalecido las concepciones tradicionales de género, que adjudican a las mujeres, las labores de atención y cuidado en la esfera privada como su función "natural" y como "expresiones de amor" identifica como condición de género; por tanto, en ese transcurrir a través del tiempo, la labor doméstica sigue siendo concebida, no como un trabajo, sino como una "responsabilidad" producto de la división del trabajo por género ${ }^{5}$.

Esta situación permite que no se reconozca el obvio valor económico e impide que las mujeres sean consideradas como población económicamente activa. Lo anterior sugiere la necesidad de estudiar la experiencia laboral de las mujeres, integrando su ejercicio laboral extra hogar con las tareas dentro del mismo y su función reproductiva, lo que significa el reconocimiento de una división de género, que dota a las mujeres de los roles de madre y esposa, entre otros. En México la temática del trabajo doméstico, aunque ha sido abordada, continúa, en su mayoría, bajo la misma tendencia de ser explorada independientemente del trabajo extra-doméstico ${ }^{1}$.

\section{El juego de los roles en el trabajo extradoméstico}

Dentro de las aproximaciones al estudio de la experiencia de las mujeres en el desempeño simultáneo de estos dos tipos de trabajo, se han caracterizado aquellas orientadas a evaluar el impacto sobre la salud en general y sobre la salud reproductiva en comunidades no indígenas. Guendelman y Silberg ${ }^{6}$ estudian el efecto de algunas situaciones domésticas y extradomésticas en la salud física y emocional de trabajadoras de maquilas; y Lara ${ }^{5}$ relaciona algunas condiciones domésticas y extradomésticas con el malestar emocional de prestadoras de servicios de salud, específicamente con enfermeras, identificando síntomas depresivos y tensión ocupacional por la dificultad de brindar cuidado a sus hijos, dado los turnos que desempeñan. Estudios fuera de México, como el de Martikainen ${ }^{7}$ apoyados en la formulación de hipótesis de "roles tensionantes", "acumulación de roles" y "convergencia", se han orientado a evaluar el impacto que tiene para las mujeres el desempeño de actividades dentro y fuera del hogar sobre su salud, y cómo este efecto se traduce en mejoras en la condición de salud y disminución de la mortalidad prematura.

La "hipótesis de roles tensionantes", señala que el desarrollo simultáneo de varios roles que 
desempeñan algunas mujeres, al ser esposas, madres y trabajadoras asalariadas, tiene efectos nocivos sobre la salud, ya que cada rol por sí mismo genera tensión y conflicto. Los efectos se traducen en fatiga, enfermedad, e incluso la muerte prematura, acompañados de un gasto adicional de energía y de tiempo, dada la dificultad para llevar el desempeño simultáneo de los múltiples roles, por la sobrecarga y el conflicto que se origina entre ellos ${ }^{8}$. Sin embargo la "hipótesis de acumulación de roles", coincide en los efectos negativos de esta multiplicidad de roles, pero rescata un elemento importante al señalar que los efectos negativos o nocivos pueden ser llevaderos, gracias a los efectos positivos que igualmente se producen; como los privilegios del rol, la seguridad del status, los recursos para realzar el estatus y el enriquecimiento personal y la gratificación ${ }^{9}$.

Realizar un trabajo extra-doméstico puede generar otros elementos positivos: posibilidad de alcanzar independencia financiera y económica de la pareja, generar mayores redes sociales fuera de casa y del matrimonio, aumentar la autoestima al compensar faltas o debilidades de otras esferas de la vida, y liberarse de ciertas tareas. La acumulación de roles es más gratificante que estresante, en la medida en que se presenta como fuente de salud, autoimagen positiva y prestigio ${ }^{10}$. La acumulación de diferentes roles dan sentido y significado a la vida, alcanzando un mayor bienestar emocional y evitando desarrollar conductas autodestructivas ${ }^{11}$. Modelos como los descritos han sido analizados en distintas poblaciones de mujeres pero no existen estudios que sirvan como paralelo nacional ni internacional, orientados a conocer específicamente, la experiencia de mujeres indígenas en torno a la forma en que se enfrentan a múltiples roles, como resultado de su incursión a la vida laboral fuera del hogar.

El proyecto "Casas de salud de las mujeres indígenas" resulta un buen ejemplo para analizar el papel del género en el trabajo extradoméstico de mujeres indígenas, ya que representa un espacio laboral donde actualmente participan. Es uno de los pocos esfuerzos institucionales implementados para atender los rezagos en materia de salud de estas poblaciones. Conjunta la participación de la población indígena, las organizaciones de la sociedad civil y las instituciones de salud, cuyo objetivo es servir de eje de las actividades de promoción y atención a la salud reproductiva y la violencia doméstica, que realizan las promotoras indígenas y servir como punto de enlace con los servicios formales de atención a la salud. Hasta la fecha y con financiamiento de la Comisión $\mathrm{Na}$ - cional para el Desarrollo de los Pueblos Indígenas (CDI), han sido ubicadas 5 Casas de salud en 4 estados de la república: una en Chiapas; una en Guerrero; dos en Oaxaca y una en Puebla.

En cada Casa trabajan en promedio de 6 a 8 mujeres: coordinadora (administra los recursos, gestiona vínculos con autoridades institucionales y organiza las actividades de la Casa), asesoras (brindan apoyo y orientación de las acciones que se realizan); promotoras, parteras, enfermeras y abogadas, orientadas a la atención de la salud materna y la violencia doméstica, desde el ámbito psicológico, legal y médico. El interés de la presente investigación fue identificar, a través del discurso de prestadoras de servicio de las Casas de Salud de las Mujeres Indígenas de México en Ometepec, Guerrero y Matías Romero, Oaxaca, la condición de género en el desempeño de su rol como trabajadoras.

\section{Metodología}

El presente fue un estudio cualitativo, basado en un análisis secundario, a partir de las entrevistas realizadas en las 5 casas de salud de mujeres indígenas existentes en el territorio nacional, realizadas durante 2007 a informantes clave: coordinadoras y asesoras (internas y externas), personal operativo y recursos locales de atención a la salud que colaboran en las Casas, mujeres usuarias de los servicios de la Casa y otros informantes, como parte de la investigación evaluativa realizada por el Instituto Nacional de Salud Pública $\left(\right.$ INSP) ${ }^{12}$. Para el presente estudio se retomaron las entrevistas de un total de 8 mujeres trabajadoras de Ometepec, Guerrero, y 6 mujeres de Matías Romero, Oaxaca. Estas mujeres fueron agrupadas de acuerdo al cargo desempeñado: "coordinadoras", se ubicaron las mujeres que se desempeñan como coordinadoras o asesoras de la casa, y en "personal operativo", el resto de las trabajadoras (promotoras, parteras, abogada y enfermera).

Para el análisis de las entrevistas nos auxiliamos con el programa Atlas-ti (v.5.0), mediante una aproximación interpretativa ${ }^{13}$ se identificaron nueve categorías que se definieron a partir del objetivo general del estudio. La investigación fue aprobada por los Comités de Investigación y Ética del INSP, cuidando aspectos éticos durante el levantamiento de los datos. Las mujeres autorizaron su participación en el estudio al igual que la grabación de sus entrevistas mediante cartas de consentimiento informado. 


\section{Resultados}

Los resultados se presentan de acuerdo a las 9 categorías de análisis. No se realizó diferenciación por estado ni cargo dada la similitud en los resultados o sutiles diferencias, que en su momento serán señaladas. Se presentarán en dos apartados: Satisfactores donde se identifican categorías que denotan agrado y beneficio para las mujeres; y No Satisfactores donde se incluyen categorías en donde se expresa malestar o dificultad para las informantes.

\section{Satisfactores}

Enriquecimiento y gratificación. Las informantes en su totalidad plantean sentir una alta satisfacción al poder ayudar con su trabajo a las mujeres de su comunidad. Apoyarlas a través de la capacitación, acompañarlas, escuchar sus experiencias y aconsejarlas, les produce sentimientos de bienestar, motivándolas a continuar con su tarea, pese a las dificultades económicas que enfrentan. También consideran tener una doble satisfacción, pues al ayudar a otras se ayudan a sí mismas al considerarse en riesgo de poder vivir experiencias similares por hacer parte de la misma comunidad.

En el caso de las mujeres del grupo de personal operativo, señalan que a partir del desempeño de su cargo se sienten más seguras y menos temerosas, para orientar un grupo, hablar en público y poder manejar sus relaciones familiares, caracterizadas en su mayoría, por agresiones físicas y psicológicas.

Privilegios del rol. En el caso de las coordinadoras, su rol les otorga la posibilidad de opinar y tomar decisiones, principalmente en momentos críticos de la organización. De igual manera, les permite estar en la planeación, seguimiento y evaluación de todas las actividades, y modificar, estructurar o rediseñar, de acuerdo a su criterio, muchas de las tareas que se realizan en la institución, con lo que adquieren un conocimiento amplio y completo sobre el funcionamiento de la casa de salud, incluyendo la posibilidad de estar en contacto con todos los miembros de la organización, y lograr apoyos interinstitucionales adicionales a los acuerdos inicialmente pactados. En el caso del personal operativo, los privilegios del rol se traducen en la posibilidad de influir sobre las mujeres de su comunidad, las cuales por el grado de confianza que les tienen les permiten que las aconsejen y les den información. ...porque las mujeres que ya me conocen tienen la confianza en ir y decirme -oye ¿sabes qué?, dame una orientación ¿no?, ¿cómo le hago? a ¿cómo esto?-. Por lo menos acuden a mi para un consejo... (P6: análisis Personal operativo.txt - 6:12).

Recursos y estatus. En cuanto a los recursos y el estatus, las coordinadoras evidencian como ganancias adicionales, la oportunidad de asistir a foros y colaborar en investigaciones, participar o no en actividades que les son de su agrado y tener cierta influencia sobre las demás trabajadoras, al ser identificadas como personas de gran experiencia y conocimiento. Por su parte, las mujeres del nivel operativo señalan como ganancias, el reconocimiento que han alcanzado dentro de su comunidad, la posibilidad de ayudar a otros en situaciones complejas y obtener beneficios personales, gracias al acceso que tienen a individuos con cierto nivel de influencia:

...estaba... no me acuerdo cómo se llama un doctor, y él me llenó las referencias y me dijo que él me conocía porque él iba a la casa de salud, y me dijo: -jahi tu eres una de las promotoras- sí, -le digo- y ya me dijo que me fuera con mis papeles, que con esto ya me los podrían firmar. (P4: Análisis Personal operativo.txt - 4:5).

Así mismo, los dos grupos identifican como ganancia adicional, poder asumir posiciones importantes dentro de su comunidad, a partir de ofrecimientos directos, tales como los madrinazgos de niños:

...yo me acuerdo, vino una compañera acá, pidió que la apoyara y la apoyé, y yo fui y la acompañé y todo y vino y me dice: -oye, quiero que seas madrina de mi hijo ¿no?-, -le digo -no, este, ¿cómo crees?, no, o sea, no quiero que me agradezcas de esa manera, yo no, además no puedo. Vino como cinco o seis veces, y me dice: -no, es que yo quiero que tú seas madrina de mi hijo porque, este, me salvaste, y no tengo una manera [de agradecértelo], le digo -no, no te preocupes-. Yo no quería, entonces me dijo -oye, pues, acepta... (P2: Análisis Coordinadoras.txt - 2:18).

Seguridad y estatus. De otra parte la seguridad y el estatus, se manifiestan en sentimientos mutuos de agradecimiento por las enseñanzas y apoyo para superar situaciones difíciles de tipo personal; $y$ en un sentido de solidaridad entre pares, el cual genera seguridad y funge como elemento motivador para continuar desarrollando a satisfacción las labores.

Percepción del rol. Finalmente las mujeres frente a su rol se perciben entre sí como personas con un alto sentido de compromiso, máxime si la labor que desempeñan es voluntaria y casi gra- 
tuita. Así mismo identifican que debido a que son mujeres trabajadoras con experiencias similares a las vividas por muchas de sus usuarias, pueden generar credibilidad frente a su comunidad y convertirse en ejemplo a seguir. Sin embargo el resultado es contrario cuando se evalúa la percepción que tienen de su rol aquellas instituciones con las que trabajan frecuentemente, pues identifican que para algunos funcionarios el trabajo que ellas desarrollan, no es importante, tendiendo a ser subvalorado y/o menospreciado, lo que identifican a partir de las negativas o la indiferencia ante invitaciones o citaciones.

...pero por el simple hecho nosotras nos damos cuenta que ellos no quieren, este, responder, porque cuando nosotras los invitamos [algunos funcionarios de otras instituciones] para nuestras concentraciones, para nuestros talleres, no asisten... (P1: Análisis Coordinadoras.txt - 1:5).

\section{No satisfactores}

Carencia de recursos. La carencia de recursos económicos en la institución, emerge como uno de los principales factores que generan insatisfacción en las coordinadoras y el personal operativo, debido a que la falta de un salario fijo, las obliga a desempeñarse en labores adicionales para obtener algún ingreso:

...pero cuando no hay proyecto cada quien agarra su yo vendo, yo vendo [se refiere a la actividad de vender]. Cuando estoy en mi tiempo de venta dos veces a la semana vendo y con eso nos sostenemos, y así cada quien puede seguir haciendo su labor en la casa [de salud]... (P 2: Análisis Coordinadoras.txt - 2:15).

De otra parte esta carencia de recursos, implica que se trabaje en condiciones precarias, materiales, equipos y personal de trabajo son insuficientes; e impide el desarrollo efectivo de ciertas labores, como el desplazamiento a lugares clave para su gestión (hospital y comunidades apartadas). Hacer este tipo de actividades exige, generalmente, colectas de dinero entre las trabajadoras o financiamientos personales, los cuales tienen que justificar para ser reembolsados; proceso que se dificulta dadas las condiciones en las que laboran:

...para canalizar a las mujeres embarazadas yo me voy con ellas pagando de mi dinero, de mi dinero, porque ni modo que me vaya caminando de aqui hasta el hospital regional, está a varias millas. Aquí es así, paga una de las que estamos aquí. (P 1: Análisis Coordinadoras.txt - 1:2).

Múltiples roles. Se plantea una gran dificultad al desempeñarse como trabajadoras, dadas las demás funciones que realizan fuera de la institución. La mayoría son casadas y tienen hijos por lo que requieren distribuir sus tiempos y alternar sus actividades entre el trabajo y las obligaciones propias del hogar. Por lo general trabajan durante la semana en la casa de salud, y los fines de semana, cuando no hay talleres, los destinan a labores familiares y caseras. Sin embargo, cuando no reciben salario, realizan actividades alternas que les permiten obtener ingresos para su manutención y la de sus familias. Las mujeres de nivel operativo, experimentan sentimientos de culpa relacionados con la doble función de trabajadora y madre, pues consideran estar abandonando a sus hijos por salir a realizar un trabajo, el cual no brinda un ingreso económico significativo que compense el abandono temporal del hogar:

...es muy difícil, porque, porque yo que soy madre, a veces como no tengo un salario, no tengo una compensación en la casa de salud, llego a mi casa, me siento tan triste cuando mis hijos llegan a mi y no les llevo nada y realmente pues sí duele ¿no? que yo les vaya a quitar en lugar de llevar... (P6: Análisis Personal operativo.txt - 6:17).

Obstáculos. Como principal obstáculo las informantes señalan, al igual que en carencia de recursos, la falta de un ingreso económico, que les permita asistir de manera más regular y poder realizar sus actividades con mayor efectividad.

... ha sido dificil, o sea, este, porque cuando no recibes así un apoyo, no puedes estar aquí, pues necesitas tu comida, tu hospedaje, no sé.Yo he venido a acompañar cuando puedo... (P2: Análisis Coordinadoras.txt - 2:9).

Vulnerabilidad. Las informantes aunque no manifiestan ser agredidas físicamente, sí identifican actitudes de indiferencia y apatía por parte de algunos servidores institucionales, las cuales interpretan como otra forma de agresión. El personal operativo, específicamente, refiere que por su condición de indígenas, en muchas ocasiones son discriminadas o agredidas verbalmente en las instituciones donde no las conocen, y señalan ser objeto de burlas por parte de algunos miembros de la comunidad, por laborar para una organización indígena. Así mismo existe el temor de ser agredidas o despreciadas por los hombres de su comunidad, al identificarlas como personas que están haciendo cambiar la forma de pensar de sus mujeres y con lo cual ellos podrían verse afectados:

...nos han dicho las mismas mujeres, -ustedes están bien vigiladas, quién entra, quién sale, porque ustedes nos orientan cómo poder actuar para 
salir de esto, entonces si yo demando o dejo a mi marido, contra ustedes se van a ir-... (P2: Análisis Personal operativo.txt - 2:19).

\section{Discusión}

$\mathrm{Al}$ analizar el discurso de las mujeres trabajadoras respecto a su experiencia laboral, pudimos identificar en primer lugar, la importancia que ellas le confieren al trabajo que desempeñan y el compromiso que expresan hacia su comunidad, como las principales razones que lo hacen posible, dado dos aspectos relevantes: uno, que la realización personal está desvinculada de la ganancia económica, ya que en muchos casos, o es poco significativa, o en otros, ni siquiera se recibe. Esto es relevante dadas las condiciones precarias en las que viven estas mujeres y las múltiples necesidades de tipo económico que tienen.

$\mathrm{Y}$ dos, estas mujeres se enfrentan a una serie de obstáculos en el desempeño de su trabajo, algunos de los cuales, se deben a la construcción cultural de su identidad como mujeres indígenas, que les dificulta su inserción en la vida laboral. Esto se traduce en un fuerte cuestionamiento de su actividad fuera del hogar, dado que, además de no recibir un salario que apoye los gastos familiares, hay una percepción comunitaria, que las ubica como mujeres que abandonan su hogar, desatendiendo funciones caseras y de asistencia temporal a los hijos, para realizar labores que, al favorecer las condiciones de salud e incentivar la lucha frente a la violencia intrafamiliar de su grupo de pares, son vistas como una amenaza para el contexto social en el que viven y para su núcleo familiar, especialmente para sus esposos.

Realizar un trabajo fuera del hogar, sumado a las responsabilidades que tienen como madres y esposas, es motivo de preocupación para estas mujeres quienes experimentan sentimientos de culpa por considerar que no están cumpliendo adecuadamente con su rol de género; lo cual corresponde a la hipótesis de roles tensionantes planteada por Goode ${ }^{8}$. Esta situación ya ha sido descrita en otros grupos de mujeres no indígenas. Por ejemplo, a nivel nacional se ha descrito que el cuidado de los hijos, representa una enorme carga de trabajo y es fuente de tensión. Al igual que se han identificado correlaciones muy significativas entre la tensión del rol materno, el papel con la pareja, la situación ocupacional y el conflicto entre el papel de madre y trabajadora ${ }^{14-}$ ${ }^{17}$. En el caso de las mujeres indígenas de este estudio, estos sentimientos de culpa pueden verse más acentuados por las presiones sociales y por las pocas alternativas que tienen, en el caso del cuidado de los hijos. Adicionalmente la expresión de sentimientos de culpa, vale la pena verlos como un factor diferenciador entre culturas, si bien para las indígenas y otros grupos de mujeres trabajadoras, este sentimiento respecto a los hijos, toma una fuerza importante, para otros no es así necesariamente.

Sin embargo, pese a la tensión, se identifica un conjunto de elementos que se convierte para estas mujeres, en fuente de satisfacción y de realización personal, y que actúa como compensatorio de los efectos negativos antes referidos. Este proceso corresponde a la teoría de los múltiples roles planteada por Sieber ${ }^{9}$, y que funciona como complemento a la hipótesis de Goode ${ }^{8}$, en donde uno de los principales elementos es el de la gratificación del ego; el cual para las mujeres de este estudio, se manifiesta en la posibilidad de ayudar a otras mujeres con sus mismas características y con el mismo tipo de problemática, básicamente relacionadas con la violencia, situación que les permite realizar un proceso de identificación, a través del cual podrían estar resolviendo estados internos de conflicto. Así mismo, el apoyo, los consejos, la orientación y el nivel de confianza que brindan a las mujeres de su comunidad las hace sentir importantes, ya que a través de los agradecimientos que reciben, perciben que su gestión impacta positivamente en su comunidad.

Este sentimiento de satisfacción se convierte en una fuente de motivación, que les permite sobreponerse a las adversidades relacionadas con las exigencias de su hogar y las vividas en su desempeño laboral, como la falta de recursos para realizar de manera más efectiva su trabajo, las frecuentes agresiones verbales, psicológicas o de actitud, o la carencia de un salario, como se señalaba anteriormente. Desde otro punto de vista, estas acciones de apoyo y orientación que son la base de este trabajo, podrían ser entendidas como una extensión de las que desempeña como madre y esposa, que dota a la mujer de una función "cuidadora" de otros ${ }^{18}$. El anterior elemento podría explicar igualmente, el por qué estas trabajadoras disfrutan su labor, sin demostrar dificultad para desempeñarla, pues son acciones que están fuertemente ligadas con las tareas que realizan como madres y esposas: cuidar, apoyar, orientar y servir; su gestión extrahogar no difiere en gran medida de las acciones que proveen para los miembros de su familia.

Otro elemento de bienestar identificado y también señalado en la teoría de múltiples roles 
es la seguridad que representa para estas mujeres desempeñar el cargo asignado, dado que a partir de las relaciones que en el marco institucional establecen con sus compañeras de trabajo, generan vínculos basados en el apoyo mutuo y la solidaridad, los cuales les permiten soportar situaciones difíciles y tensionantes. Este fenómeno puede explicarse mejor a partir de la perspectiva de redes sociales, la cual claramente nos habla de ese intercambio entre individuos, donde la solidaridad es el fin para prestar ayuda y servir como soporte moral ${ }^{19}$. De manera que esta nueva red social que las mujeres establecen a partir de su trabajo, las llena de un beneficio que no solo les permite, gracias a ese apoyo, sobreponerse a las dificultades, sino que emerge como un motivador constante y un reforzador positivo respecto a la labor que desempeñan, y con ello supone un efecto directo sobre la autoestima de cada mujer. La permanencia de las mujeres que trabajan en la casa hace que esta red sea además, estable.

Los privilegios y el estatus que las mujeres alcanzan con su trabajo, se convierten en las dos últimas fuentes de bienestar identificadas a partir de este estudio. Su importancia radica en el papel motivador y de satisfacción que representan y se traducen en prestigio y reconocimiento, a partir de los cuales estas trabajadoras tienen la posibilidad, tanto en su contexto laboral como social, de opinar, influir, orientar, asesorar, decidir, y acceder a ciertos privilegios que mejoran sus condiciones de vida y/o de las personas cercanas a ellas. Lo anterior puede tener una significancia importante si se tiene en cuenta la carencia de prebendas y las pocas posibilidades de participación que tiene la mujer indígena dentro de su sistema social ${ }^{2}$; de manera que tener un papel activo dentro de sus comunidades, haciéndole frente a problemáticas compartidas por una gran mayoría de mujeres, puede entenderse, como la oportunidad de tener el poder y el control, en un contexto donde el poder y el control casi nunca están en manos de la mujer.

$\mathrm{Al}$ respecto es claro el proceso de empoderamiento que han comenzado estas mujeres al empezar a tener control sobre algunos aspectos de su vida, aun cuando también queda claro que para poder alcanzar un verdadero empoderamiento tendrán que tener cierto grado de participación en las estructuras de poder o ingerencia en la toma de decisiones ${ }^{20}$, lo cual aún no se ha alcanzado e implica para ellas un importante camino por recorrer. En este proceso, las mujeres se enfrentan principalmente a las exigencias de un medio masculinizado, en donde la mayoría de figuras de poder son hombres, quienes tienden a minimizar e ignorar la gestión que realizan. Estas actitudes masculinas podrían interpretarse como temor a compartir espacios hasta ahora dominados por el sexo masculino o temor a aceptar la gestión de estas mujeres, pues significaría avalar, temas como la violencia, la cual es propiciada en gran medida por sus congéneres. Así las burlas, las evasiones y el maltrato verbal y psicológico, acompañado ocasionalmente de amenazas directas o veladas, que las atemoricen o las lleven a abandonar su tarea, reflejan en el fondo temor a ser confrontados con sus propios esquemas culturales, pero sobretodo a tener que confrontarse con su mismo grupo de pares.

Centrándonos en la teoría de múltiples roles, podríamos señalar que para este estudio se cumplen muchos de sus planteamientos. Los resultados en términos generales, son equiparables a los resultados encontrados en investigaciones con otros grupos de mujeres no indígenas trabajadoras, como el realizado por Martikainen ${ }^{7}$, en donde claramente se evidencia la presencia de elementos compensatorios que actúan como amortiguadores de los efectos negativos del desempeño de varios roles. Esto es interesante si se tiene en cuenta que las poblaciones de estudio distan notablemente de poseer las mismas características, salvo el de ser mujeres. En el estudio de Martikainen ${ }^{7} y$ en investigaciones realizadas por otros autores, las mujeres estudiadas no pertenecen a contextos indígenas, lo que representa un hallazgo valioso del presente estudio, en la medida en que estas comunidades tienen unas características socioculturales muy específicas y diferenciadoras de otras.

Lo anterior sugiere, que pese al contexto, las mujeres trabajadoras presentan tensiones relacionadas con la estructura social y cultural en la que viven; pero la participación en varios roles puede hacer que uno de ellos mitigue los efectos adversos de otros ${ }^{21}$, generando beneficios que les pueden a ayudar no solo a aligerar los conflictos, sino a permitirles disfrutar y continuar en su ejercicio laboral. Una interpretación a esto obedecería a que comparten ciertas características del rol de género. La reproducción, la crianza, el cuidado y el desarrollo de las labores domésticas han sido atribuidas culturalmente a las mujeres en diferentes sociedades ${ }^{22,23}$. Así mismo su incursión en el ámbito laboral, ya sea por necesidad económica o por deseo de realización personal, se ha realizado de manera gradual y ha significado un proceso difícil y complejo, en unas sociedades más que en otras, dependiendo del contexto ${ }^{22}$. Es 
importante tener en cuenta que la presencia de elementos compensatorios en distintos grupos de mujeres (indígenas y no indígenas), no necesariamente se traduce en iguales contenidos significativos, el mismo proceso, ni igual jerarquía. Por ejemplo, cuando nos referimos a la gratificación como elemento que genera ganancia a todas las mujeres trabajadoras, debemos tener presente que este fenómeno se da de manera diferente en las mujeres indígenas, respecto a las mujeres de contextos no indígenas.

Para mujeres no indígenas, el dinero, por ejemplo, puede ser motivo de gratificación; éste puede hacerles sentir importantes, adicionalmente porque les implica adquirir cierta independencia de sus esposos; mientras que para las mujeres indígenas el dinero puede no tener el mismo significa- do, son otros elementos los que para estas comunidades adquieren más valor y significancia, como el reconocimiento a partir de labores que favorecen las condiciones de sus congéneres, las cuales son las que les producen alta satisfacción. No podemos perder de vista que estas mujeres viven en condiciones de pobreza, por tanto el dinero podría ser importante, pero dada su poca accesibilidad, se confiere mayor significancia a otros aspectos de la vida que les son más cercanos. La experiencia laboral de las mujeres trabajadoras en la "casa de la mujer indígena" de Matías Romero, Oaxaca, y Ometepec, Guerrero, descrita en este trabajo muestra que tiene características muy específicas de difícil replicación en otras regiones del país. En estudios futuros es necesario considerar los diferentes contextos de vulnerabilidad.

\section{Colaboradores}

C Amaya-Castellanos y Blanca Pelcastre-Villafuerte: concepción y delineamiento, análisis e interpretación de los datos, redacción del artículo y revisión crítica, aprobación de la versión a ser publicada. M Márquez-Serrano: participación en la redacción del artículo, revisión crítica y la aprobación de la versión a ser publicada. C Juárez Ramírez: revisión crítica y la aprobación de la versión a ser publicada. 


\section{Referencias}

1. García B, Blanco M, Pacheco E. Género y trabajo extradoméstico. En: García B, coordinadora. $\mathrm{Mu}$ jer, género y población en México. México DF: El Colegio de México, Sociedad Mexicana de Demografía; 1999. p. 273-316.

2. Comisión Nacional para el Desarrollo de los Pueblos Indígenas. [Sitio de la Internet]. [Consultado 2008 ene 23]. Disponible en: http://www.cdi.gob.mx/ index.php?id_seccion $=609$

3. Fernández-Ham P, Embriz-Osorio A, Serrano-Carreto E, Medina-Domínguez ME. Características económicas. En: Ham PF. Indicadores con perspectiva de género para los pueblos indígenas. México DF: Comisión Nacional para el Desarrollo de los Pueblos Indígenas; 2006. p. 127-149.

4. Lamas M. El género. La construcción cultural de la diferencia sexual. México DF: Universidad Nacional Autónoma de México; 1996.

5. Lara M. Investigación sobre trabajo femenino y salud: avances y propuestas. En: Figueroa J, compilador. La condición de la mujer en el espacio de la salud. México DF: El Colegio de México; 1998. p. 131-160.

6. Guendelman S, Silberg JM. The health consequences of maquiladora work: women on the US-Mexican border. Am J Public Health 1993; 83(1):37-44.

7. Martikainen P. Women's employment, marriage, motherhood and mortality: a test of the multiple role and role accumulation hypotheses. Soc Sci Med 1995; 40(2):199-212.

8. Goode WJ. A theory of role strain. Am Sociol Rev 1960; 25(4):483-496.

9. Sieber SD. Toward a theory of role accumulation. Am Sociol Rev 1974; 39(4):567-578.

10. Marks SR. Multiples roles and role strain: some notes on human energy, time and commitment. Am Sociol Rev 1977; 42(6):921-936.

11. Thoits PA. Multiple identities and psychological wellbeing: a reformulation and test of the social isolation hypothesis. Am Sociol Rev 1983; 48(2):174-187.

12. Pelcastre B, Márquez M, Meneses S, Ruiz M, Amaya C, Gómez Y. Proyecto Rescatando la experiencia de la "casa de la mujer indígena": sistematización y evaluación del proceso. CONACYT (SALUD-2005-0214445). Cuernavaca: Instituto Nacional de Salud Pública; 2006.

13. Denzin N. Un punto de vista interpretativo. En: Denman C, Haro JA, compilador. Por los rincones. Antología de métodos cualitativos en la investigación social. México: El Colegio de Sonora; 2000. p. 147-205.
14. Lara MA, Acevedo M, López EK, Fernández M. La salud emocional y las tensiones asociadas con los papeles de género en las madres que trabajan y en las que no trabajan. Salud Mental 1993; 16(2):13-23.

15. Garduño M, Saldaña J. Salud y doble jornada: taquilleras del metro. Salud Problema 1990; 20:41-45.

16. Hoffman L. Effects of maternal employment in the two parent family. Am Psychol 1989; 44(2):283-292.

17. Márquez M, Romero J. El desgaste en las obreras de la maquila eléctricoelectrónica. Salud Problema 1988; 14:9-24

18. Tezoquipa-Hernández I, Arenas-Monreal LM, Valdez-Santiago R. El cuidado a la salud en el ámbito doméstico: interacción social y vida cotidiana. Rev Saude Publica 2001; 35(5):443-450.

19. Bronfman M. Como se vive se muere: familia, redes sociales y muerte infantil. México: Universidad Nacional Autónoma de México, Centro Regional de Investigaciones Multidisciplinarias; 2001:143-166.

20. Rowlands J. Empoderamiento y mujeres rurales en Honduras: un modelo para el desarrollo. En: León M, compiladora. Poder y empoderamiento de las mujeres. Bogotá: Universidad Nacional de Colombia; 1997. p. 213-244.

21. Kandel D, Davies M, Raveis V. The stressfulness of daily social roles for women: marital, occupational and household roles. J Health Soc Behav 1985; 26(1): 64-78.

22. Programa de las Naciones Unidas para el Desarrollo (PNUD), Instituto de Pesquisa Económica Aplicada do Brasil (IPEA). Relatorio sobre o desenvolvimiento humano no Brasil, 1996, Brasilia: Programa de las Naciones Unidas para el Desarrollo (PNUD).

23. Instituto de Pesquisa Econômica Aplicada do Brasil (IPEA); 1996

Rindfuss RR, Brewster KL, Kavee AL. Women, work, and children: behavioural and attitudinal change in the United States. Pop Dev Rev 1996; 22(3):457482 .

Artigo apresentado em 09/06/2010

Aprovado em 05/11/2010

Versão final aprovada em 30/11/2010 